\title{
Revisited "Cluster-Cluster" VLBI with future multi-beam low frequency radio interferometers
}

\author{
María J. Rioja* ${ }^{\dagger}$ and Richard Dodson \\ ICRAR, UWA, Perth, Australia \\ E-mail: maria.rioja@uwa.edu.au, richard.dodson@uwa.edu.au
}

Richard W. Porcas

Max-Planck-Institut für Radioastronomie, Bonn, Germany

E-mail: porcas@mpifr-bonn.mpg.de

Dick Ferris and John Reynolds

ATNF, CSIRO, Sydney, Australia

E-mail: Dick.Ferris@atnf.csiro.au, John.Reynolds@csiro.au

\section{Tetsuo Sasao}

E-mail: sasaotts@nifty.com

\section{Richard T. Schilizzi}

SKA Program Development Office, The University of Manchester, UK

E-mail: schilizzilskatelescope.org

We revisit the "Cluster-Cluster" or multi-view VLBI technique from the perspective of its synergy with the multi-beam features inherent in the Australian Square Kilometer Array Pathfinder (ASKAP) and its potential to improve the outcomes of VLBI observations with ASKAP. We include a list of candidate VLBI sites that already support or can be upgraded to support multiview VLBI located in Australia and overseas, and which have common visibility with ASKAP. The results of our previous cluster-cluster observations at $1.6 \mathrm{GHz}$ demonstrated the advantages of this configuration to calibrate the ionospheric distortions responsible for the loss of positional accuracy at low frequencies, using multiple calibrators in a range between 1 to 6 degrees away from the target. Therefore, we conclude that joint observations of ASKAP with other multi-view sites using cluster-cluster techniques would improve the outcomes of the high spatial resolution component of ASKAP applied to astrometric projects, achieving higher precision for many more targets, and with lower detection thresholds. Also very wide-field VLBI mapping becomes a possibility. Looking to the future, this would contribute to the development of new techniques that are relevant for future high resolution observations with the SKA.

Science and Technology of Long Baseline Real-Time Interferometry:

The 8th International e-VLBI Workshop, EXPReSO9

June 22 - 262009

Madrid, Spain

\footnotetext{
*Speaker.

$\dagger$ On secondment from OAN, Spain.
} 


\section{Introduction}

Conventional Very Long Baseline Interferometry (VLBI) phase-referencing techniques can achieve the highest precision relative astrometric measurements of a target source, using alternating observations of a nearby calibrator source, at frequencies between a few and tens of GHz. At lower frequencies, i.e., below $2 \mathrm{GHz}$, its application is constrained. The cause of this limitation is the unpredictability of both the temporal variations and the spatial irregularities in the plasma density in the ionosphere, which introduce differential path variations between the observations of the two sources, even for very fast switching times and small source separations. The temporal and spatial differential ionospheric fluctuations degrade the positional accuracy of the technique and, eventually, prevent the phase connection process and the use of conventional phase referencing. These fluctuations are larger at lower frequencies.

In general, observations which involve more than one calibrator have demonstrated advantages for astrometric VLBI at low frequencies. The exception is the ideal but unusual configuration when a target and a strong calibrator lie within the field-of-view (FoV) of the VLBI antennas (an "in-beam" calibrator), and thus can be observed simultaneously. A useful variation of this combines the observations of an "in-beam" weak calibrator source and nodding to a more distant strong calibrator. The observations of the strong calibrator are used to remove the first-order atmospheric effects; then the observations of the weak source, which is observed along with the target source, provide further adjustments (a fine tuning) of the spatial and temporal fluctuations, with longer coherence times [2]. The results obtained with this approach are promising; however its widespread application is still limited by sensitivity. Another useful approach is when there are two calibrators aligned with, but on opposite sides of, the target. During the observations the telescopes alternate every few minutes between the three sources, and in the analysis successive scans on the calibrators are used for the spatial and temporal interpolation to the enclosed position and scan time of the target source [1]. The unlikely source configuration required for this approach to work results in limited applicability, and the overhead calibration time is large.

This contribution is concerned with the multi-view or cluster-cluster VLBI technique, which allows simultaneous observations of a target and multiple calibrators around it by replacing single telescopes by sites with multiple elements. The suitability of this technique to address the ionospheric effects has been demonstrated with joint observations between connected interferometer arrays at $1.6 \mathrm{GHz}$ of a target and three calibrator sources (see Fig. 1). Despite these benefits its use has been limited by the shortage of observing sites, and the complexity in its implementation. We wish to revisit this technique now in the light of the next generation of instruments for low frequency observations that will become operational in the course of the next decade, for which the multi-beam capability is an "in-built" feature, such as ASKAP in the near future, and SKA in the long term. We believe that the implementation of cluster-cluster techniques will enhance the performance of VLBI observations with ASKAP by providing higher precision astrometric measurements of many more targets, along with an extremely wide-field mapping capability. 


\section{2. “Cluster-Cluster" VLBI: Basics and Demonstration}

Cluster-Cluster or multi-view VLBI is a technique that replaces single-beam telescopes by sites with multiple-beam capabilities (a "cluster") [7]. The cluster-cluster concept offers the prospect of correcting for phase perturbations arising from the spatial and temporal structure in the troposphere and ionosphere, since such structure can be modelled using simultaneous observations of multiple reference sources. The basic requirements for a site to conduct cluster-cluster observations are that the elements (usually antennas) are fed from a common frequency standard, be equipped with a precise phase calibration system and have the ability for independent pointing. This ability makes cluster sites flexible to adapt the sensitivity of the observations, by adapting the number of antennas allocated to the observations of each source.

The feasibility and advantages of this approach have been demonstrated with observations, for example, carried out in November 1999 with 3 interferometers, the VLA, WSRT and MERLIN, at $1.6 \mathrm{GHz}$ (see [3-6] for a detailed description of all our observations). Each interferometer observed four sources simultaneously, selected from the VLBA calibrator list such that one of them, the target, lies in the sky surrounded by the other three sources, the calibrators. The angular separations from the central target source were 1,3 and 6 degrees, as shown in Figure 1. We carried out a comparative analysis of techniques which aimed to correct the ionospheric distortion of the wavefront, that is, using a combination of the observations on the three calibrators, and using a single calibrator at a time. The combined analysis showed that a simple 2-D linear interpolation of the ionospheric paths would be sufficient to correct the phase of the target source at the central position, using the phases measured on the three other sources. Moreover, the 2-D linear model derived from the simultaneous observations of the three sources resulted in a superior phase compensation compared to that from the (phase) difference between each of the pairs to the central source, as done in conventional phase referencing. Thus, our results demonstrated the superiority of the cluster-cluster technique to compensate the ionospheric errors in VLBI observations, even with respect to a single calibrator only 1 degree away, at $1.6 \mathrm{GHz}$.

\section{ASKAP in the context of "Cluster-Cluster" VLBI}

ASKAP is a next generation mid-to-low-frequency radio interferometer and one of the SKA demonstration telescopes, located in Western Australia. It comprises 36 12-m multi-beam antennas distributed over $6 \mathrm{~km}$, with most of them lying within a circle $2 \mathrm{~km}$ in diameter. It incorporates novel receiver technology consisting of phased-array-feeds and beam forming modules, which will result in an extremely wide FoV of about 30 square degrees in the observing frequency band from 0.7 to $1.8 \mathrm{GHz}$, using about 30 beams per antenna. It will observe an instantaneous bandwidth equal to $300 \mathrm{MHz}$ and 2 polarizations.

ASKAP will participate in VLBI observations as a tied-array, and will retain the unique multibeam feature. The number of planned tied-array beams will be at least equal to that of antenna beams, and fully steerable within the wide antenna FoV. In other words, ASKAP operating in VLBI mode will be equivalent to having about 30 single-beam antennas, each with a collecting 
area equivalent to a 64-m dish, and independent pointing within the wide ASKAP FoV of about 30 square degrees. Hence, joint VLBI observations with a network of conventional single-beam antennas, with typical FoVs of about 0.5 to 1 degree, would only make use of a small fraction of the capabilities of ASKAP.

ASKAP meets the criteria to be a cluster, with the steerable multiple tied-array beams taking the place of the steerable single-beam antennas. To implement VLBI cluster-cluster techniques it is vital that the multi-beam operations of ASKAP can be matched with similar multi-view capabilities from other instruments which have common visibility with ASKAP. This is a list of candidate VLBI sites that already support or can be upgraded to support multi-view VLBI located in Australia and overseas. In Australia, the Australia Telescope Compact Array (ATCA), with 6 22-m antennas, the Parkes Observatory, with a 64-m antenna and a 12-m antenna, both of which will be equipped with multi-beam receivers, and the Mount Pleasant Radio Observatory, with three independent antennas; plus overseas, the Giant Meter-wave Radio Telescope (GMRT), in India, and MeerKAT, South Africa's SKA pathfinder (see Fig. 2).

We are working towards testing the feasibility of VLBI observations with a cluster-cluster network of Australian sites. Such a network would provide the necessary counterpart to exploit the full capabilities of ASKAP in the VLBI domain.

\section{Discussion}

ASKAP fulfills the conditions to be a cluster site, and a key element for cluster-cluster VLBI observations due to its high sensitivity, which results from the large collecting area and wideband recording, and the wide FoV. The results of our cluster-cluster experiment, with observations of a target surrounded by a group of three calibrators distributed across a region equivalent to the wide ASKAP FoV, demonstrated the advantages of this configuration to calibrate the ionospheric distortions, which degrade the positional accuracy in conventional "nodding" VLBI observations at low frequencies. ASKAP will be located in Western Australia, which happens to be a part of the world with common visibility with other VLBI sites which hold the potential to carry out multiview VLBI. There are plans to study the needs to upgrade these candidate sites to be clusters and the feasibility of joint observations with ASKAP. Three of the sites are members of the Australian VLBI network (LBA) and routinely carry out joint observations. Other candidate sites are located overseas, in India and Africa. In addition to those, any single-beam antenna site could become a cluster if equipped with a phased-array-feed receiver. Therefore, we conclude that joint observations of ASKAP with other multi-view sites using cluster-cluster techniques would improve the outcomes of ASKAP applied to astrometric VLBI measurements, achieving higher precision for many more, and a broader range of, targets, and with lower detection thresholds for imaging of weak sources. Also, cluster-cluster techniques would export the extremely wide FoV capability of ASKAP as a stand-alone instrument to VLBI observations with ASKAP, by enabling multiple wide-field VLBI mapping.

We discuss here the feasibility of VLBI astrometry at low frequencies, based on the probability of finding suitable calibrators, using ASKAP either with a VLBI array of clusters or with 
a single-beam antenna network. Best astrometry with a single-beam antenna network is carried out using phase referencing techniques with an "in-beam" calibrator, or the variation of this which is a weak "in-beam" calibrator plus nodding. The application of the "in-beam" technique, nevertheless, is constrained by the small probability of finding such a calibrator within the same FoV, about 1 square degree, as the target. For a given target, the probability would be higher the greater the sensitivity of the array. On the other hand, the cluster-cluster technique achieves comparable astrometric results with the additional advantage that it can draw calibrators from a larger fraction of the sky, as wide as the FoV of ASKAP, about 30 square degrees. For comparison, we have done a rough estimate of the impact of the increased sensitivity and wide FoV on the number of astrometric calibrators, in observations of ASKAP with a network of single-beam antennas and a network of clusters, respectively. The number of "in-beam" calibrator sources, required for astrometry with ASKAP in a VLBI array of single-beam antennas, will grow with the increased recorded bandwidth to the power of approximately 0.85 , since the sensitivity is proportional to the square root of the bandwidth and assuming a $\log N-\log S$ source distribution has an index of about -1.7 . Hence a bandwidth increase by a factor of 10, which corresponds to an upgrade from the current LBA standard mode to the bandwidth of ASKAP, would increase by about 7 times the number of "in-beam" calibrators. In turn, the number of cluster-cluster calibrator sources for astrometry with ASKAP in a network of multi-view sites will scale with the FoV, which is about 30 times more. The conclusion is that there are more sources to be had from an expanded FoV than from an increased bandwidth. Therefore, from the perspective of available calibrators, the cluster-cluster technique has a more comprehensive application. The two techniques are not exclusive, and their combined use would lead to the optimal outcome, which can be judged on a case-by-case basis.

Looking to the future, the ASKAP is a pathfinder for the SKA Program, and one of the next generation telescopes that will see light in the coming decade. The outlined benefits of using cluster-cluster techniques in VLBI observations with ASKAP would apply equally to some of those instruments. Ultimately, establishing a cluster-cluster network for VLBI observations with ASKAP would contribute to developing new techniques that are relevant for future high resolution observations with the SKA in the mid-frequency range, and exploit the technological development for the SKA to improve the scientific capabilities of VLBI.

\section{References}

[1] Fomalont, E.B., Goss, W.M., Beasley, A.J., Chatterjee, S., Sub-Milliarcsecond Precision of Pulsar Motions: Using In-Beam Calibrators with the VLBA, 1999, AJ, 117, 3025

[2] Fomalont, E.B., Kopeikin, S. Phase Referencing Using Several Calibrator Sources, 2002, Proc. 6th European VLBI Network Symposium, 53.

[3] Porcas, R.W., Alef, W., Rioja, M.J., Desmurs, J-F., Gurvits, L. \& Schilizzi, R., Multi-view VLBI with Arrays in Cluster-Cluster Mode, 2003, Proc. IVS Symp. "New Technologies in VLBI" (2002), ASP Conf. Ser. 306, p 39-51

[4] Rioja, M.J., et al., A Cluster-Cluster VLBI experiment, 1995, Proc. 10th Working Meeting on European VLBI for Geodesy and Astrometry. 


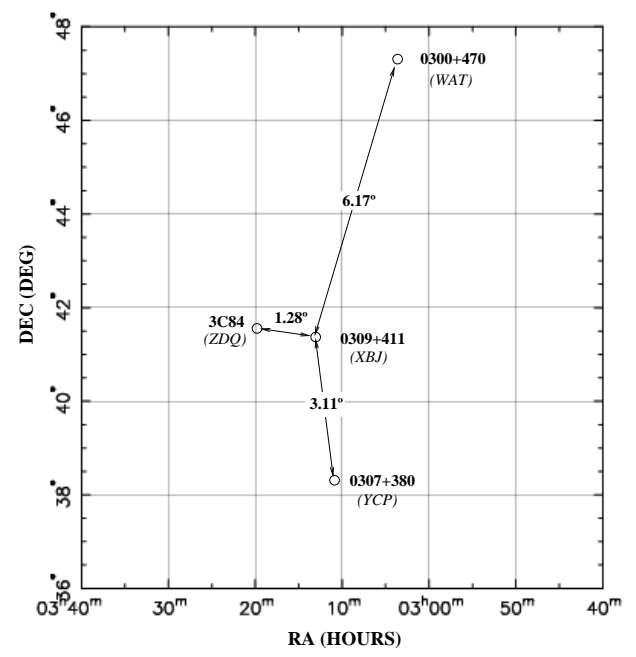

Figure 1: Sky distribution of the sources simultaneously observed in our cluster-cluster observations carried out in 1999 with VLA, WSRT and MERLIN interferometers, at $1.6 \mathrm{GHz}$. The sources, strong and compact, belong to the VLBA calibrator list. The idea behind this technique is that the observations of a target source, in the middle, are calibrated using a combination of the phase measurements on the other three sources.

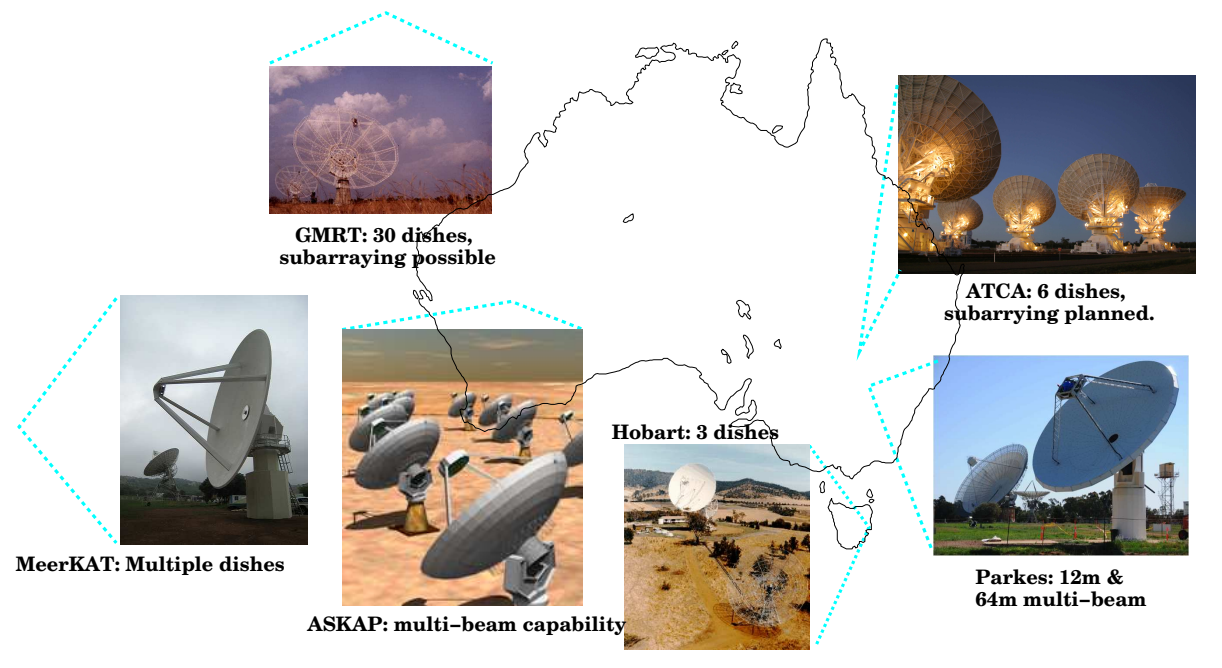

Figure 2: Map of potential multi-view facilities in Australia and overseas for joint VLBI cluster-cluster observations with ASKAP.

[5] Rioja, M. J., Stevens, E., Gurvits, L., Alef, W., Schilizzi, R. T., Sasao, T., Asaki, Y., Phase-referencing in Cluster-Cluster VLBI mode, 1997, Vistas in Astronomy 41, 213.

[6] Rioja, M. J., Porcas, R. W., Desmurs, J.-F., Alef, W., Gurvits, L. I., Schilizzi, R. T., VLBI observations in Cluster-Cluster mode at 1.6 GHz., 2002, Proc. 6th EVN Symposium, 57.

[7] Sasao, T., Morimoto, M., 1991, Proc. of the Chapman Conference on Geodetic VLBI: Monitoring Global Change, NOAA Tech. Rep. NOS 137 NGS 49, 48. 